\title{
APPLICATION \\ OF STATISTICAL SPIN LIQUID CONCEPT TO HIGH-TEMPERATURE SUPERCONDUCTIVITY
}

\author{
K. Byczuk and J. Spalek \\ Institute of Theoretical Physics, Warsaw University \\ Hoża 69, 00-681 Warszawa, Poland
}

\begin{abstract}
We apply the concept of statistical spin liquid, in which the doubly occupied quasimomentum configurations $\{|k \uparrow \downarrow\rangle\}$ for quasiparticles are excluded from the Fock space, to a planar superconductor with real space pairing. The results compare very well with experimental data for the cuprates, namely (i) the condensed state appears only for the number of holes $0<\delta<0.15-0.25$; (ii) the temperature dependence of the gap is close to the BCS result, but the gap has systematically lower value and is of an extended $s$-wave form.

PACS numbers: 71.27.+a, 74.20.Mn
\end{abstract}

Experimental data suggest that the high-temperature superconductors in the normal state cannot be regarded as conventional metals. Transport properties are difficult to describe within the Landau Fermi-liquid theory [1]. Also, a smooth evolution of the magnetic susceptibility from that of a metal to that of a magnetic insulator points to a spin liquid character of the normal state [2]. The specific-heat and the Hall constant measurements indicate that as the system undergoes the gradual transition to the insulating state the effective mass does not diverge, but instead the carrier density approaches zero [3]. Simultaneously, photoemission experiments confirm the existence of the Fermi surface in the metallic regime [4].

We have recently proposed [5] a novel approach to strongly correlated metals which would reconcile the unexpected experimental facts with the quasiparticle picture of electrons in the solids. The treatment is based on the fundamental observation that when the value of the Coulomb repulsion energy exceeds by far the width of the quasiparticle band, the doubly occupied single-spin configuration must be excluded not only in real space, but also in reciprocal $(k)$ space. In other words, the electrons can only move in the lower Hubbard subband [6], whereas the double occupied $k$ states $\{|\boldsymbol{k} \uparrow \downarrow\rangle\}$ appear only as resonant states leading to the Cooper pairing instability below a critical temperature [7]. Above that temperature we expect also a non-Fermi liquid behaviour of the imaginary part of the self-energy with the inverse relaxation time proportional to temperature $\left(\tau^{-1} \sim T\right)[7]$. These resonant states from the upper subband would diminish the volume enclosed by 
the Fermi sphere and hence, the Luttinger theorem is obeyed. Also, the assurnption of the double occupied state exclusion in reciprocal space allows for a continuous transformation of correlated itinerant states into the Mott insulating state with a band filling $n \rightarrow 1$. In the insulator regime the high temperature entropy is exactly $k_{\mathrm{B}} \ln 2$ per carrier [5], as one should have expected for the Mott insulator, when only the spin degrees of freedom are important. This continuous evolution of electron liquid into the magnetic insulator provides the reason for calling the itinerant state a statistical spin liquid (SSL).

In this paper we present only the results for the superconducting state of SSL. Normal state properties will be discussed elsewhere [7].

We start with the $t-J$ Hamiltonian [8] transformed into the reciprocal space and projected onto the space with the doubly occupied states $\{|k \uparrow \downarrow\rangle\}$ excluded. The form of the model Hamiltonian is then

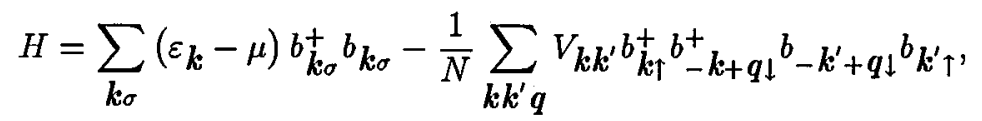

with $\varepsilon_{\boldsymbol{k}}$ being the bare band energy of electrons; $V_{\boldsymbol{k} k^{\prime}}=4 \varepsilon_{k_{k}} \varepsilon_{k^{\prime}} / U$, with $U$ being the magnitude of the inraatomic Coulomb interaction between electrons with opposite spins, and $b_{k_{\sigma}}^{+} \equiv a_{k_{\sigma}}^{+}\left(1-n_{k_{\bar{\sigma}}}\right)$ is the creation operator of a singly occupied quasiparticle state $|k \sigma\rangle$ in the lower Hubbard subband. Due to the projections these operators obey nonfermion anticommutation relations of the form

$$
\left\{b_{k_{\sigma}}, b_{k^{\prime} \sigma^{\prime}}^{+}\right\}=\delta_{k k^{\prime}}\left[\left(1-n_{k \bar{\sigma}}\right) \delta_{\sigma \sigma^{\prime}}+b_{k_{\bar{\sigma}}}^{+} b_{k \sigma}\left(1-\delta_{\sigma \sigma^{\prime}}\right)\right],
$$

with $n_{k_{\sigma}}=a_{k_{\sigma}}^{+} a_{k_{\sigma}}$.

We have solved this model in a mean field approximation for the commutator Green function [9] and obtained the following self-consistent equations for the gap parameter $\Delta_{k}$ in the paired state, and for the chemical potential $\mu$ :

$$
\Delta_{k}=\left(1-\frac{\bar{n}_{k}}{2}\right) \frac{1}{N} \sum_{k^{\prime}} V_{k k^{\prime}} \frac{\Delta_{k^{\prime}} \tanh \left(\frac{\beta}{2} E_{k^{\prime}}\right)}{2 E_{k^{\prime}}}
$$

and

$$
n=\frac{1}{N} \sum_{k} \bar{n}_{k}=\frac{1}{N} \sum_{k} \frac{2 v_{k}^{2}}{1+v_{k}^{2}},
$$

where

$$
v_{k}^{2} \equiv \frac{1}{2}\left[1-\frac{\varepsilon_{k}-\mu}{E_{k}} \tanh \left(\frac{\beta}{2} E_{k}\right)\right],
$$

and $E_{k} \equiv\left[\left(\varepsilon_{k}-\mu\right)^{2}+\left|\Delta_{k}\right|^{2}\right]^{1 / 2}$. From (3) we obtain the $k$ dependence of the gap in the form: $\Delta_{k}=\left(1-\bar{n}_{k} / 2\right) \varepsilon_{k} \Delta(T)$. Apart from the statistical factor, the encrgy gap has an extended $s$-wave symmetry; this is caused by the inclusion of three-site hopping processes in the $t-J$ Hamiltonian in direct space [10]. The statistical factor $\left(1-\bar{n}_{k} / 2\right)$ is equal to $2 / 3$ at the Fermi level, lowering the value of the gap in the quasiparticle spectrum near and below the Fermi level. Also, the presence of this factor leads to an asymmetry of the density of states shape in the near gap regime; such feature may be detected in tunneling experiments. 
The particle number equation (4) is of the same type as in the normal phase case [5] ( $v_{k}^{2}$ plays the role of the Fermi-Dirac distribution in superconducting state); indicating the non-Fermi liquid character of electrons in the lower Hubbard subband. These equations were solved numerically for a square-lattice superconductor. In Fig. 1 we have plotted the superconducting transition temperature $T_{\mathbf{c}}$ as a function of hole number $\delta=1-n$ for different values of $U$ (measured in the units of $2|t|$, where $|t|$ is the value of the nearest neighbor hopping). The common feature of our solutions is a disappearance of $T_{\mathrm{c}}$ in the insulator limit $(\delta=0)$, as well as for $\delta \sim 0.15-0.25$; this is in excellent agreement with the experimental

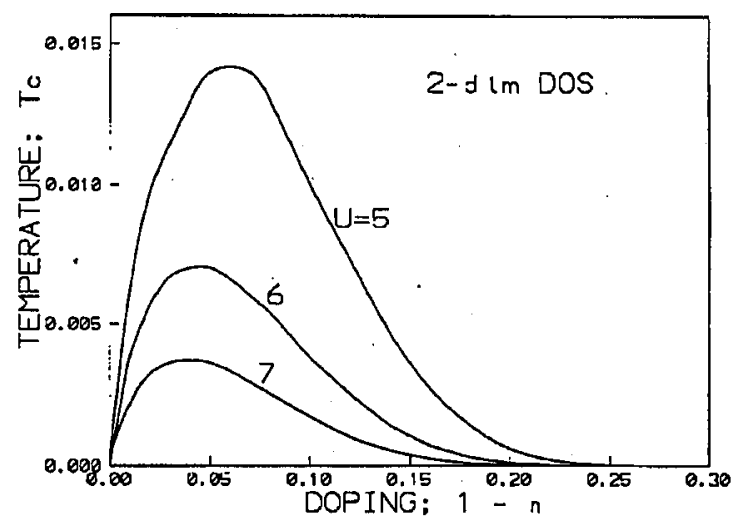

Fig. 1. Band filling dependence of the transition temperature; the values of $U$ are in units of $2|t|$ (spin liquid $t-J$ model).

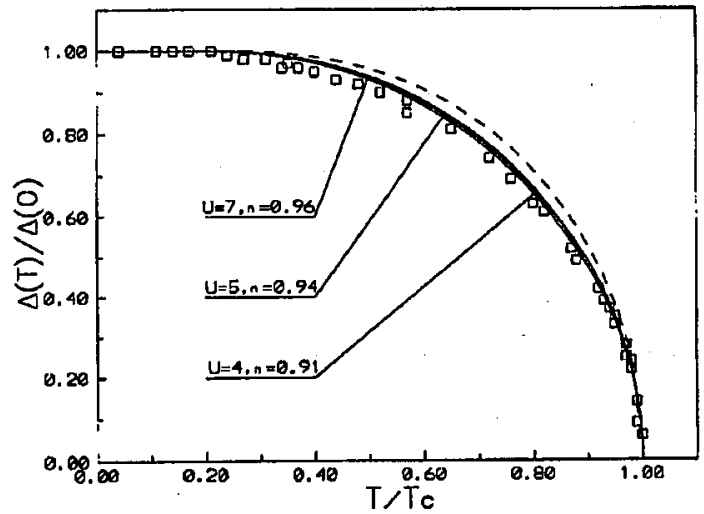

Fig. 2. The reduced gap parameter $\Delta(T) / \Delta(0)$ versus reduced temperature $T / T_{\mathrm{c}}$ (solid lines); the BCS result (dashed line) is displayed for comparison. The squares represent the recent experimental results taken from Ref. [12]. 
phase diagram for $\mathrm{La}_{2-x} \mathrm{Sr}_{x} \mathrm{CuO}_{4}$ [11]. Similar behavior was obtained for $\Delta(0)$. The ratio $2 \Delta_{k_{\mathrm{F}}} / k_{\mathrm{B}} T_{\mathrm{c}}$ is about 3.3 , a value lower than the $\mathrm{BCS}$ value 3.53 ; this is due to the presence of the statistical factor $\left(1-\bar{n}_{k} / 2\right)$ in $\Delta_{k}$.

We calculated also the temperature dependence of the gap parameter $\Delta=\Delta(T)$. The relative gap $\Delta(T) / \Delta(0)$ versus the ratio $T / T_{\mathrm{c}}$ is plotted in Fig. 2, where we compare our theoretical results (solid lines) with those from BCS theory (dashed line), as well as with the very recent experimental data [12] for the in-plane component of $\Delta_{k}$ (squares). The agreement is excellent. Our results have a systematically lower value than the corresponding numbers in BCS theory which is based on the Fermi-liquid approach.

To summarize briefly, we applied the SSL concept to the superconducting state and have obtained a very good agreement with the experimental data for the cuprates.

The authors acknowledge the grants from the Committee for Scientific Research of Poland. J.S. was also partially supported by the MISCON grant No. DE-FG 02-90ER 45427.

\section{References}

[1] See e.g. M.F. Crommie, G. Briceno, A. Zettl, Physica C 162- 164, 1397 (1990).

[2] J.M. Tranquada, in: Earlier and Recent Aspects of Superconductivity, Eds. J.G. Bednorz, K.A. Müller, S rringer, Berlin 1990, p. 422.

[3] T. Nishikawa, S. Shamoto, M. Sera, M. Sato, S. Ohsugi, Y. Kitaoka, K. Asayama, Physica C 209, 553 (1993).

[4] G.G. Olson, R. Liu, A.-B. Yang, D.W. Lynch, A.J. Arko, R.S. List, B.W. Veal, Y.C. Chang, P.Z. Jiang, A.P. Paulikas, Science 245, 731 (1989).

[5] J. Spalek, W. Wójcik, Phys. Rev. B 37, 1532 (1988); J. Spałek, Phys. Rev. B 40, 5180 (1989); Physica B 163, 621 (1990); J. Spałek, W. Wójcik, Sci. Bull. Jagellonian University 29, 87 (1990).

[6] J. Hubbard, Proc. R. Soc. Lond. A 281, 401 (1964).

[7] K. Byczuk, J. Spalek, in preparation.

[8] K.A. Chao, J. Spałek, A.M. Oles', J. Phys. C 10, L271 (1977).

[9] K. Byczuk, J. Spałek, submitted to Phys. Rev. B; see also J. Spalek, K. Byczuk, J. Karbowski, W. Wójcik, Phys. Scr., in press.

[10] J. Spałek, Phys. Rev. B 38, 533 (1988).

[11] J.B.Torrance, Y. Tokura, A.I. Nazzal, A. Bezinge, T.C. Huang, S.S.P. Parkin, Phys. Rev. Lett. 61, 1127 (1988).

[12] E. Polturak, G. Koren, D. Cohen, E. Aharoni, Phys. Rev. B 47, 5270 (1993). 